\title{
A Variational Approach to Design a Numerical Scheme for N-Fluid Flow
}

\author{
Vazquez-Gonzalez Thibaud, Llor Antoine \\ CEA, DAM, DIF 91297 Arpajon Cedex, France \\ thibaud.vazquez-gonzalez@cea.fr; antoine.llor@cea.fr
}

\section{Extended Abstract}

In some highly demanding fluid dynamics simulations, it appears necessary to simulate multiphase flows involving numerous constraints at the same time, such as: large number of fluids (typically 10 and above), both isentropic and strongly shocked compressible evolution, large heat sources, large deformations, transport over large distances, and highly variable or contrasted equation of state stiffenesses. Fulfilling such a challenge in a robust and tractable way demands that thermodynamic consistency of the numerical scheme be carefully ensured $[1,2]$. This is addressed here over an arbitrarily evolving computational grid (ALE or Arbitrary Lagrangian-Eulerian approach) by a three-step mimicking derivation [3]:

i) to ensure a compatible exchange between kinetic and internal energies under isentropic conditions, a variational least action principle is used to generate the proper pressure forces in the momentum equations; ii) to generate the conservative internal energy equation, a tally is performed to match the kinetic energy, and iii) artificial dissipation is added to ensure shock stability, but other physical terms could also be included (drag, heat exchange, gravity, etc.).

The resulting multiphase scheme named multiGEECS (Geometry, Energy, and Entropy Compatible multiphase Schemes) involves the following features: i) full conservation of mass, momentum, and total energy of the system at discrete level (up to round-off errors); ii) direct ALE formalism where mass, momentum, and internal energy fluxes are taken into account directly into the discrete evolution equations (without separation between Lagrangian evolution and remapping procedure); iii) thermodynamic consistency of the pressure work obtained by application of a variational principle; iv) pressure equilibrium through a simple and local (to the cells) procedure; and v) generic set of evolution equations written for an arbitrary number of fluids and derived without any constraint on structure or spatial dimension in order to simulate a broad category of multiphase flows.

Multiphase numerical test cases are performed in two-dimensions using various strenuous grid motion strategies. The results confirm the following properties: i) exact conservation at discrete level (proper capture of shock levels and shock velocities); ii) robust multi-material like behavior with small residual volume fractions; iii) stable multiphase behavior where each fluid has its own velocity in order to obtain drifting between fluids; iv) preservation of isentropic flows; and v) versatility regarding grid motions (including supersonic shearing, linear interpolation of contact discontinuity, nearLagrangian motions, or randomly distorted mesh). 

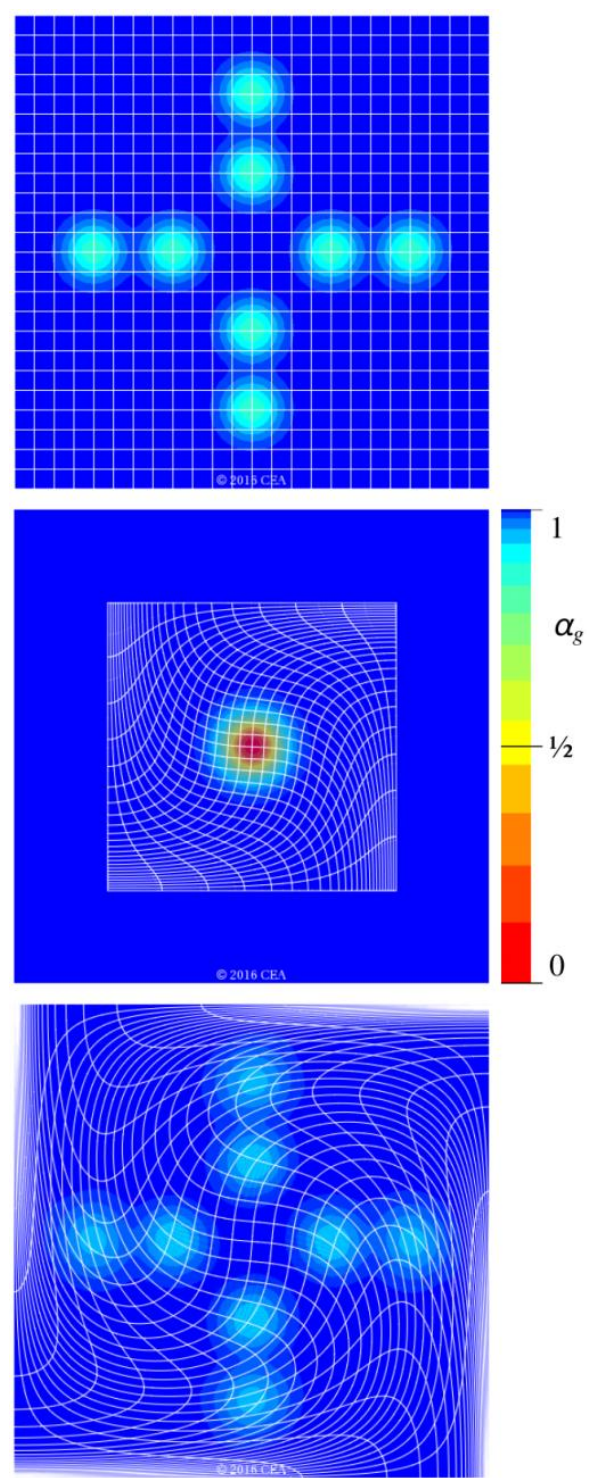

Fig. 1: Crossing of eight packets of heavy fluids in a surrounding light gas. Heavy fluids are described by a stiffened gas EOS and the light fluid is described by a perfect gas EOS. Initial packets close-to-sonic velocities are chosen so that all packets cross at the center of the domain. In order to demonstrate the stability and robustness of the scheme, the computation are carried out on a shrink-then-stretch swirling ALE grid. Every displayed macro cell (white lines) corresponds to 20x20 numerical cells for a total of 480x480 cells.

\section{References}

[1] T. Vazquez-Gonzalez, A. Llor, C. Fochesato, "Ransom test results from various two-fluid schemes: is enforcing hyperbolicity a thermodynamically consistent option?," Int. J. Multiphase Flow, vol. 81, pp. 104-112, 2016.

[2] A. Llor, A. Claisse, C. Fochesato, "Energy preservation and entropy in Lagrangian space-and-time-staggered hydrodynamic schemes," J. Comput. Phys., vol. 309, pp. 324-349, 2016.

[3] T. Vazquez-Gonzalez, A. Llor, C. Fochesato, "A novel GEEC (Geometry, Energy, and Entropy Compatible) procedure applied to a staggered direct-ALE scheme for hydrodynamics," Eur. J. Mech. B-Fluids, vol. 65, pp. 494514, 2017. 